\title{
Fixkombination überzeugt beim Aknemanagement
}

— Die Deutschen Akneleitlinien empfehlen mit hohem Evidenzlevel als Standardbehandlung bei entzündlicher Akne u.a. ein topisches Retinoid (wie Adapalen) und/oder eine antimikrobielle Substanz wie Benzoylperoxid (BPO). Dass sich internationale und nationale Experten für eine Kombinationsbehandlung im Rahmen des Aknemanagements aussprechen, hat gute Gründe: Die Wirkung einer Fixkombination aus Adapalen $0,1 \%$ und $\mathrm{BPO} 2,5 \%$ (Epiduo $^{\circledR}$ ) erwies sich in zwei klinischen Studien mit insgesamt über 2.00o Aknepatienten bei guter Verträglichkeit als signifikant besser als die Monotherapien mit den jeweiligen Einzelsubstanzen oder dem Vehikel [J Am Acad Dermatol 2007; 57: 791-9; Br J Dermatol 2009; 161: 1180-9]. Unter dem Einfluss der Zweierkombination bildeten sich in den Studien innerhalb von zwölf Wochen entzündliche Läsionen um $62 \%$ und nichtentzündliche Läsionen um $54 \%$ zurück. Auch im Vergleich zu topischen Fixkombinationen aus BPO und dem topischen An-

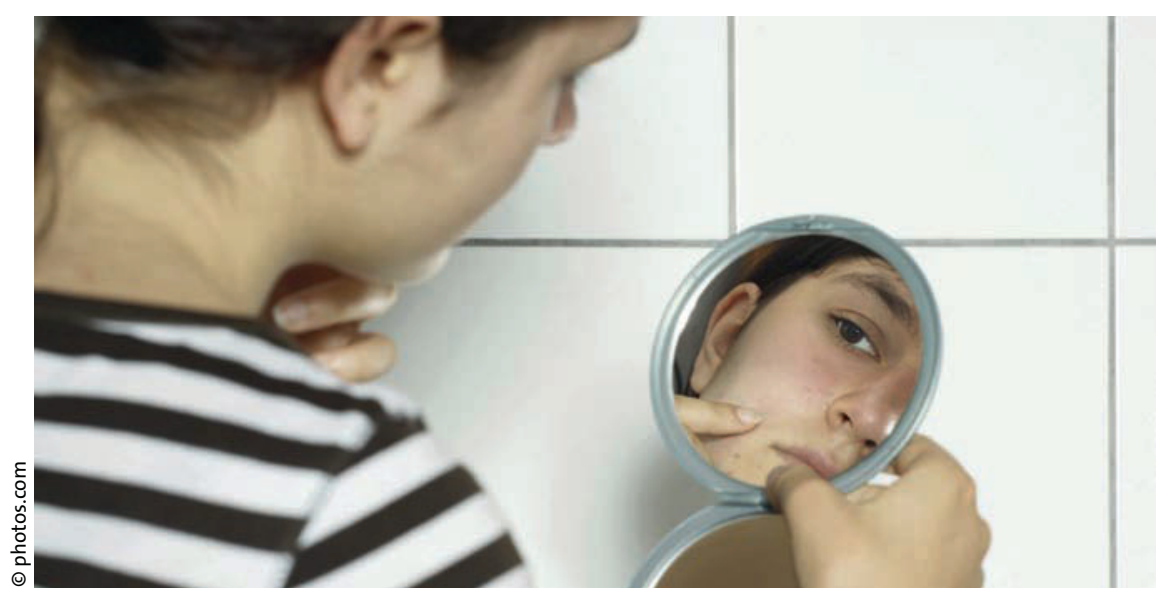

Schon nach wenigen Tagen können Aknepatienten mit der Fixkombination Erfolge verzeichnen.

\section{Wundheilung durch natürliche Komponenten}

\section{- Mit Actrys ${ }^{\circledR}$ steht ein Wundheilmittel} zur Verfügung, das vier natürliche Elemente beinhaltet: Ton, Honig, Wachs und Öle, die reich an essenziellen Fettsäuren sind. Angewendet werden kann Actrys ${ }^{\circledR}$ bei Dekubitus des Stadiums II, III und IV, Geschwüre an den Beinen, postoperativen Narben, varikösen Geschwüren und nicht zuheilenden Wunden.

Ton hat die Funktion, Druck auf die Wundränder auszuüben. Dieser Druck trägt zur Hämostase bei und folglich auch zur Bildung von Fibrin. Ton hat außerdem eine starke Absorptionsfähigkeit für Flüssigkeit. Auf diese Weise werden die Exsudate und andere Wundsekrete aufgesaugt. Zusätzlich adsorbiert Ton pathogene Keime und verhindert damit ihre Proliferation. Die
Schichtstruktur des Tons ermöglich die Einlagerung von Ölen. Die Öle, die reich an essenziellen Fettsäuren sind, sind natürliche Aktivatoren der Zellregeneration. Das ebenfalls in dem Wundmittel enthaltene Wachs bildet einen Schutzfilm, der die Wunde einerseits vor Verunreinigungen von außen schützt und andererseits einen Feuchtigkeitsverlust der Wunde verhindert. Honig wiederum besitzt entzündungsfördernde Eigenschaften. Aufgrund seines hohen Gehalts an Polysacchariden aktiviert und stimuliert Honig die Entzündungsreaktion und damit das Phänomen der natürlichen Wundreinigung. Außerdem wir die Proliferation von Bakterien durch den sauren ph-Wert von Honig, der zwischen 5,2 und 5,6 liegt, gehemmt. Dank seines tibiotikum Clindamycin zeigte sich, dass die Fixkombination Epiduo ${ }^{\circledR}$ auch ohne Antibiotikum gleich stark wirksam ist [Therapeutics for the Clinician 2009, 84: 1-7]. Lassen sich diese guten Ergebnisse auch in der täglichen Anwendung in der Praxis reproduzieren? Eine aktuelle, offene, nicht-interventionelle Studie, die unter Alltagsbedingungen bei Patienten in Dermatologiezentren durchgeführt wurde, bestätigt die Ergebnisse der großen klinischen Zulassungsstudien: Die antibiotikafreie Fixkombination aus Adapalen und BPO mindert äußerst effektiv entzündliche Läsionen und ist dabei gut verträglich. 91 mit Epiduo ${ }^{\circledR}$ behandelte Patienten (15 Jahre oder älter) mit leichter bis mittelschwerer Akne wurden in die Untersuchung aufgenommen. Während des zwölfwöchigen Behandlungszeitraums verminderte sich die mittlere Anzahl der entzündlichen Läsionen um $80,6 \%$ und die der nicht-entzündlichen um 69,3\% ( $p<0,001)$. Die Effektivität der Medikation spiegelte sich auch in der globalen Beurteilung der Wirksamkeit durch die Ärzte wider: Der IGA-Score verminderte sich von ursprünglich 2,9 (moderat schwere Akne) auf 1,1 (beinahe reine Haut) am Studienende $(p<0,001)$. Die Fixkombination wurde zudem gut vertragen.

red

Nach Informationen von Galderma

hohen Zuckergehalts stellt Honig eine Energiequelle dar, die entscheidend zur Reifung junger Zellen und damit auch zum Wundheilungsprozess beiträgt.

Durch die verschiedenen Komponenten beschleunigt Actrys ${ }^{\circledR}$ den Prozess der Wundheilung und wirkt frühzeitig ab der Hämostasephase auf die Wunde ein. Die natürliche Immunantwort wird aktiviert und die Gewebereparatur im Zentrum der Wunde stimuliert. Außerdem werden das bakterielle Ökosystem und die Exsudate unter Beachtung des Prinzips der Aufrechterhaltung eines feuchten Milieus kontrolliert. Das Wundmittel sorgt deshalb täglich dafür, dass die Wunde Schicht um Schicht verschlossen wird, bis die Gewebereparatur vollständig ist.

red 